\title{
Proceedings of the International Consensus Conference on Breast Cancer Risk, Genetics, \& Risk Management, April, 2007
}

\author{
Gordon F. Schwartz, MD, Kevin S. Hughes, MD, Henry T. Lynch, MD, Carol J. Fabian, MD, \\ lan S. Fentiman, MD, Mark E. Robson, MD, Susan M. Domchek, MD, Lynn C. Hartmann, MD, \\ Roland Holland, MD, PhD, David J. Winchester, MD, and The Consensus Conference \\ Committee*
}

\begin{abstract}
A consensus conference including thirty experts was held in April, 2007, to discuss risk factors for breast cancer and their management. Four categories of risk were outlined, from breast cancer "average" through "very high" risk, the latter including individuals with high penetrance BRCA1 / 2 gene mutations. Guidelines for management of patients in each of these categories were discussed, with the major portion of the conference being devoted to individuals with BRCA1 / 2 mutations. Prevalence of these mutations in the general populations was estimated to be 1 in 250 500 individuals, with an increased prevalence in Ashkenazic Jews and other founder groups. Risk reduction strategies for these individuals include surveillance, with or without chemoprevention drugs, or surgical procedures to remove the organs at risk, i.e., bilateral mastectomy and / or bilateral salpingo-oophorectomy. These risk reduction strategies were evaluated fully, and recommendations were made for the care of patients in each of the risk categories. These guidelines for patient care were approved by the entire group of experts.
\end{abstract}

\section{Keywords}

BRCA; breast cancer; genetics; risk

\section{Introduction}

Since the identification of the first breast cancer autosomal dominant gene in 1990, the molecular world of breast cancer investigation has exploded. Of the approximately 184,450 invasive breast cancers expected in the USA in 2008, between 5\% and 10\% resulted from highly penetrant cancer susceptibility genes. Few of these individuals were known mutation carriers before the diagnosis, when this knowledge might have led to prevention or an earlier diagnosis. Moreover, many of these cancers were not subsequently identified as "hereditary" when this knowledge might have changed their management or helped other family members still at risk.

To address these issues of risk management and prevention, the sixth international consensus conference of the Breast Health Institute was convened in Philadelphia, April 26 through April 29, 2007, inviting a group of experts in breast cancer care, cancer genetics and risk assessment, representing each of the disciplines involved in the care of these patients. The goal was to summarize the state of the art in terms of identification of patients at high risk and to discuss the various management strategies appropriate to level or type of risk. These proceedings were dis- tilled from the recorded transcripts of the entire meeting,

Address correspondence and reprint requests to: Gordon F. Schwartz, MD, MBA, FACS, Jefferson Medical College, 1015 Chestnut Street, Suite 510, Philadelphia, PA 19107-4305, USA, or gordonschwartz@yahoo.com. 
summarizing the opinions and conclusions of the entire group. Each participant has approved this manuscript; any major differences of opinion among them are noted.

\section{What Does the Term Increased Risk Mean?}

Although the "average" American woman may have a one in eight lifetime risk of breast cancer, she is also likely to have at least one or more of the so- called minor risk factors. As breast cancer incidence also varies by age, many risk counselors try to give women both a relative short term and long term estimation of age-matched risk. Risk should be expressed, however, in absolute terms, and also as compared to an "average" woman in the patient's age group (relative risk or RR).

A "risk factor" means any variable that increases the risk of breast cancer for affected individuals. Major risk factors are usually regarded as those that at least double risk whereas minor factors increase RR by between 1.00 and 2.00 (Table 1). For many major risk factors such as a BRCA1 or BRCA2 mutation, contralateral breast cancer, prior ductal carcinoma in situ (DCIS), lobular carcinoma in situ (LCIS), atypical ductal hyperplasia (ADH), atypical lobular hyperplasia (ALH), both absolute and RR are known (Tables $2 \& 3$ ). However, for many minor risk factors, we are only able to pro- vide RR estimates. Major risk factors tend to dwarf minor risk factors in individual risk assessment. For a comprehensive risk assessment, information should be sought about all of the factors in Table 1; however, once a woman has a known factor that increases her risk significantly, such as atypical hyperplasia or a genetic mutation, knowing age at menarche or first live birth adds little.

\section{Family History as a Starting Point for Risk Assessment}

The importance of a detailed family history cannot be overemphasized. The panel agreed that current time constraints in most health care systems make a comprehensive family history difficult to record. The best approach suggested by the panel was to emphasize the importance of family history to all clinicians, and to encourage the development of the electronic medical record as the best tool to collect and record such data.

Every medical record should have a family history section that should include the patient's own medical history, including cancer, past or present, and the cancer history of the progeny, siblings, parents, together with second degree relatives and both sets of grandparents. The medical histories of these older first and second generation relatives may be highly informative, as they will have passed through the cancer-risk age. A cancer history collected through three generations is usually sufficient, but in cancer-prone families (e.g., Li-Fraumeni), this should be extended as far as possible because diverse cancers may occur.

\section{Other Factors}

\section{Breast Density}

An increase in mammographically measured breast density has been recently considered by some investigators as a risk factor in its own right. The panel considered breast density as a possible risk factor, but they were frustrated by the lack of evidence-based data on which this conclusion has been reached. The breast imagers among the group, especially, were breast density has been measured. The panel concluded that currently there is no reproducible, quantifiable manner to measure breast density to be able to answer this important question. That this question must be pursued by appropriate clinical trials was not challenged, but the current publicity that has sur- rounded this assertion was disputed. 


\section{Cytological Atypia in Intraductal Fluid (Ductal Lavage)}

Whether the finding of "atypia" in a sample of intraductal fluid obtained by ductal lavage or by specialized devices should lead to the same screening recommendations as for those women found to have ADH or ALH in a surgical breast biopsy has been debated. Although FDA approved for patient use, the panel unanimously felt that these techniques should still be considered quasi-experimental and used only in the context of a clinical trial.

\section{Risk Models}

Several risk assessment models have been developed which give both short and long term risk estimates and / or the risk of mutation in BRCA1 and BRCA2. The modified Gail model is the most popular in the United States but considers only seven variables - race, current age, age at menarche, age at first live birth, number of affected first-degree relatives, number of breast biopsies, and whether the biopsy contained atypia (http://www.cancer.gov/ bcrisktool). Women who have risk factors not considered by the model will have their risk underestimated, but it is currently the model approved by the FDA in the USA for determining who should be offered tamoxifen or raloxifene (see infra). Other such models are available and widely used, especially abroad; each has its avid proponents and challengers; none is perfect.

\section{Risk Categories}

The panelists agreed to four basic categories of risk:

1. Average risk - the female population at large;

2. Moderate risk - those women whose risk is more than average for their age group but their RR is less than fivefold;

3. High Risk - more than fivefold but less than 10- fold RR for age group. This includes women with LCIS, ADH, and ALH, and many women with two firstdegree relatives with breast cancer but no mutation;

4. Very high risk - greater than 10-fold increase in RR due to either a high penetrance gene mutation (BRCA1 / 2, PTEN or Tp53) or those likely to harbor such a mutation, or a personal history of irradiation to breast or chest wall prior to age 30 , such as for Hodgkin disease.

\section{Management Strategies by Risk Category}

Because none of the risk models is perfect, how they may be used is arbitrary. It is perhaps preferable to discuss approaches to risk in a more generic manner, trying to place women in various groups, based upon their perceived risk of developing breast cancer. Therefore, approaches for different risk groups were discussed individually, with the major focus on women with likely or proven high penetrance germline mutations responsible for hereditary breast cancer. However, reasonable and applicable recommendations were proposed for each of the groups.

\section{Average Risk: (Absence of any of the Accepted Risk Factors)}

These women should follow accepted programs for cancer screening. This implies annual mammography starting at the age customary in that country (e.g., age 40 in the USA, 50 in the UK, etc). Several (American) members of the group favored starting annual mammography at age 35 . The panel did not achieve consensus on when to stop mammography, but most agreed that, if the patient had a reason- able life expectancy ( $>5$ years), mammography should continue indefinitely. Breast (and gynecologic) examinations 
are recommended also at annual intervals. Breast self-examination, although often deprecated as effective, still should be carried out monthly. Screening for other cancers, e.g., colon, should also follow accepted population-based guidelines.

Prevention strategies with tamoxifen or raloxifene are not indicated in these women, but healthy life- styles should be stressed. Menopausal estrogen therapy (HRT) for symptomatic women without a uterus was considered safe, for up to at least 10 years. Despite the current controversy about the breast cancer risk of combined HRT, the panel agreed that there was mini- mal risk in using combined HRT for up to 5 years to address quality-of-life issues.

\section{Moderate Risk: (One or more of the "minor" Factors in Table 1)}

Screening should be the same as above for the "average" risk. No data support more frequent screening or screening with magnetic resonance imaging (MRI) in this group. However, physicians should acquaint their premenopausal patients with the data about tamoxifen, and postmenopausal women about tamoxifen and raloxifene. The group did not strongly support the use of these drugs in this population but did recommend that the information about them be discussed with patients. Hormone replacement is not contraindicated for symptomatic women in this risk category, but women with an intact uterus needing combined estrogen plus a progestin should use the lowest possible dose for the shortest period of time.

\section{High Risk}

For women at high risk primarily as a result of a diagnosis of atypical ductal or lobular hyperplasia or lobular carcinoma in situ, risk of invasive carcinoma is increased at least fivefold, and annual digital mammography was advised from the date of diagnosis, irrespective of age. There are little data regarding the use of annual screening MRI in addition to mammography. The high cost of MRI and the false positive rate must be weighed against the potential for finding additional early cancers. The group felt that this was a fertile area for future study.

Semiannual clinical breast examination and symptom review were recommended. These women generally are candidates for chemoprevention with tamoxifen if premenopausal, or with tamoxifen or raloxifene if postmenopausal. However, there is no current evidence that tamoxifen or raloxifene given for primary prevention increases survival. Use of aromatase inhibitors in postmenopausal women for primary prevention is not (yet) indicated, pending results of ongoing phase III trials.

For women at high risk primarily because of multiple affected family members but without a BRCA1 or 2 mutation, screening recommendations are based on the level of risk. If lifetime risk of breast cancer due predominately to family history exceeds $20 \%$, annual MRI in addition to annual mammography is recommended. Screening should begin at least 10 years prior to the age of diagnosis of the youngest affected close relative, or by age 40, whichever is earliest. Clinical breast exam is suggested twice yearly. Consideration of chemoprevention should be based on level of risk and anticipated benefit to risk ratio, but at least should be discussed. Risk-reduction surgery, while a subject for discussion, is not a usual recommendation for these patients.

\section{Very High Risk}

This group includes a small group of women who underwent chest or breast radiation before age 30 (e.g., treatment for Hodgkin disease) or who are known to have or likely to have a deleterious mutation in a high penetrance gene responsible for hereditary breast cancer. Women with chest or breast radiation before age 30 need risk counseling and annual 
(digital) mammography beginning 5-10 years after the age of their radiation treatment or at age 40 , whichever is earlier, but not before age 25 . Annual breast MRI should be considered in these women, alternating at 6 month intervals with mammography, starting at the same age, although there is little clinical trial data to support this recommendation and no data to suggest that periodic MRI affects survival. Semiannual clinical examination starting at the same time was recommended. Whether these women should be advised to consider tamoxifen or raloxifene was more controversial, but a majority of the group thought that these would be appropriate choices to reduce risk. Risk-reduction surgery should be discussed, but like group 3 above, is not a usual recommendation. Women at very high risk due to a BRCA1 or BRCA2 mutation or similar high penetrance genes responsible for hereditary breast cancer will be discussed in detail for the remainder of this paper.

\section{Identification of Women at Hereditary Risk}

The discovery of germline mutations that increase breast cancer risk has led to greater attention being paid to these factors to determine an individual's life- time risk and to examine techniques to decrease that risk. In general, genetic factors have been divided into two arbitrary categories, namely, those of high and low penetrance.

\section{Low Penetrance Genes}

The list of low penetrance genetic markers is lengthy and growing. Each of these mutations probably confers only a small to moderate increase in the lifetime breast cancer risk.

However, because mutations in these low penetrance genes are expected in a large number of people, the population attributable risk (PAR) for breast cancer explained by these genes may be actually substantial and (potentially) considerably higher than the PAR caused by much less frequently encountered mutations of high penetrance genes such as BRCA1 and BRCA2. Lesser penetrance genes may partly account for the difference in the sensitivity of women to environmental factors, such as the use of alcohol or replacement hormones. If the interaction of environmental factors and low penetrance gene mutations can be elucidated, specific prevention strategies may become possible. This research on low penetrance genes is still in its infancy, and routine clinical testing for low penetrance gene mutations (such as CHEK2) was not recommended by the panel.

\section{High Penetrance Genes}

Mutations in BRCA1, BRCA2, PTEN, and Tp53 cause some of the highly penetrant hereditary breast cancer syndromes. These are tumor suppressor genes, creating a protein that repairs DNA thereby preventing carcinogenesis. Every cell in mutation carriers lacks one functional allele; the tumor suppressor function of that gene is lost, favoring the development of cancer. Although each of these syndromes expresses a difference spectrum of cancers, they share a general set of characteristics in the family history profile. The family histories tend to show an unusually large number of relatives with cancers (e.g., three or more with breast cancers, or two or more with ovarian cancers), young age at diagnosis, multiple cancers in a single individual (e.g., bilateral breast or breast plus ovary in one relative), and / or unusual cancers (e.g., male breast cancer) or some common cancers, e.g., prostate and pancreas, often at earlier ages.

Despite public misconceptions, high penetrance hereditary breast cancer accounts for only $5-10 \%$ of all breast cancers. However, a disproportionately high number of young women are affected. Recognition of women at risk before they develop cancer is important, to effect strategies to decrease the morbidity and mortality of cancer for these individuals. 
Li-Fraumeni Syndrome and the Tp53 Gene-Li-Fraumeni syndrome is an autosomal dominant disorder, caused by mutations in the Tp53 gene. This syndrome is characterized by an increased risk of soft tissue and osteosarcomas, leukemias, brain tumors, adrenocortical carcinomas, and breast cancers. The risk of developing breast cancer before the age of 45 is 18 times higher for females carrying this mutation as compared to the general population. The RR is greatest below the age of 20 and declines with increasing age (RR for breast cancer after the age of $45=1.8$ ). Germline mutations in the Tp53 gene probably account for less than $1 \%$ of breast cancer cases.

Cowden Syndrome and the PTEN Gene-Cowden syndrome is an autosomal dominant disorder, caused by a mutation in the PTEN gene, characterized by the development of hamartomas and benign tumors. Mutations in the PTEN gene are evident in $80 \%$ of Cowden syndrome families; these are associated with an increased risk of cancer. Female mutation carriers are believed to have a $25-50 \%$ lifetime breast cancer risk, although the data to support this level of risk is not robust. In sporadic breast cancers, it is not currently known whether PTEN plays a role in breast cancer susceptibility.

BRCA1 / BRCA2-Although modestly different in their characteristics, these two high penetrance mutations are commonly considered together as their similarities outweigh their differences. Both are considered tumor suppressor genes and are those for which commercially available testing is available. The ability to test for these mutations has led to an explosion of knowledge about hereditary cancer.

Mutations in BRCA1 and BRCA2 markedly increase the risk of both breast (at least 60\% lifetime risk) and ovarian cancer (15-40\% lifetime risk) and are responsible for about $45 \%$ of families with multiple cases of breast cancer and up to $90 \%$ of families with both breast and ovarian cancer. BRCA1 carriers are of younger age, their breast cancers are more likely to be of high grade, of medullary histology, and

"Triple Negative" [not expressing estrogen receptors / progesterone receptors (ER / PR) and human epidermal growth factor receptor 2 (HER-2)]. These women also have a much higher risk of ovarian cancer. Breast cancers in BRCA2 carriers are more likely ER / PR positive. In addition, male carriers with either mutation seem to be at an increased risk for carcinoma of the prostate as well as breast, modestly increased with the BRCA1 mutation but probably at least double the lifetime risk for carriers of the BRCA2 mutation.

The role of these mutations in the development of DCIS and the significance of relatives with DCIS are uncertain. Therefore, the panel separated these two entities, i.e., invasive cancer and DCIS, so that all of the discussion and ensuing recommendations apply to invasive cancer only. However, the nuances of difference between invasive cancer and DCIS should probably be ignored as physicians ponder whom to send for genetic counseling; they should be counted equivalently.

Current mutation screening methods vary in their sensitivity. DNA sequence analysis was considered by the panelists to be the most sensitive method of detecting unknown mutations and is commercially available. For certain populations, such as Ashkenazim, limited testing for ethnic-specific alleles may be appropriate as a first step. If positive, full sequencing is not continued. This is faster and more cost-effective. As many as $90 \%$ of the mutations found in the Ashkenazim are one of these ethnic-specific alleles, so that decisions on proceeding to full sequencing should consider the details of family history, the possibility of a variant of uncertain significance (VUS), and cost. Many published estimates of the prevalence of mutations in this gene have been derived from studies that have used methods of gene analysis other than direct sequencing, but discussion of the pros and cons and the 
results of these other techniques were beyond the scope of the conference. The panelists agreed that genetic testing for clinical use should only be performed by approved laboratories, regulated in the USA by the Clinical Lab- oratory Improvement Amendments of 1988, Public Law 100-578.

Genetic testing results may identify a deleterious mutation known to cause hereditary breast and / or ovarian cancer or the absence of such a mutation. Even if there is a known family mutation, a tested but negative individual is not at hereditary risk. Management should still depend upon the family history.

Approximately $10-15 \%$ of all tested individuals will have a VUS, which may cause substantial problems in counseling, cancer risk estimation and risk management. Clinical management of such patients needs to be highly individualized and consider factors such as the patient's personal and family cancer his- tory, as well as the likelihood that the VUS is significant. Additional research will certainly lead to a reclassification of many VUS, which may then change the care of affected individuals. The presence of a VUS is usually reported by the testing laboratory, often with a disclaimer as to its significance.

If a deleterious mutation is identified, genetic testing should be encouraged for all at-risk family members, after full counseling and obtaining informed consent. Mutation carriers should begin specialized screening, and noncarriers may revert to general population screening guidelines. Currently, any contact with relatives must be through the known mutation carrier (the patient undergoing the testing). The physician's legal responsibilities to the patient's family members when a mutation is identified have not been clearly delineated, once the index patient has been informed of the findings. While it might be ideal for the physician to inform the carrier's first- and second- degree relatives, legal action has been initiated against physicians who have given this information to relatives without the patient's express permission. Unambiguous, clear legislative decisions are needed to define the limits of the "duty to warn" family members and the most acceptable means of fulfilling that duty within the constraints of legislation (e.g., HIPAA) guarding patient privacy.

The panel agreed that genetic testing in children was indicated only with the Li Fraumeni syndrome. Most panelists saw little benefit to BRCA testing before age 25 .

Prevalence of BRCA1 and BRCA2 Mutations-The panel estimated the frequency of these mutations as about 1 per 250-500 individuals in the non- Jewish population. The younger the index patient and the stronger the family history, the higher is the likelihood of a mutation. Up to $20-25 \%$ of women diagnosed before age 30 are carriers, as are women with more than three first-or second-degree relatives with breast cancer. Ovarian cancer in a first-degree relative is also associated with a $25 \%$ chance of a BRCA1 mutation, and up to $40 \%$ if that same relative had both breast and ovarian cancer.

Sometimes the same mutation appears in multiple unrelated families, when a contemporary population derives from a small, isolated group of "founders." Two BRCA1 mutations (185delAG and 5382insC) and a BRCA2 mutation (6174delT) have been reported in Ashkenazi Jews (those tracing their roots to Central and Eastern Europe). Carrier frequencies for these mutations Ashkenazi Jewish population are approximately $1 \%$ for the 185delAG mutation, less than $0.5 \%$ for the 5382insC mutation, and $1 \%$ for the BRCA2 6174delT mutation. Altogether, the frequency of these three mutations approximates 1 in 40 among Ashkenazi Jews, accounting for $25 \%$ of early- onset breast cancer, and up to $90 \%$ of the families with multiple cases of both breast and ovarian cancer. 
Additional founder mutations have been described in the Netherlands, the French Canadian population, Ice- land, and Sweden. Additionally, there are so-called "cancer families," with significant histories of breast and / or ovarian and other cancers that warrant genetic testing, but they test negative for either BRCA1 or BRCA2 or a VUS. Although not treated in the same manner as mutation carriers, these families are at significantly increased risk for cancer, and management recommendations should be individualized based on family history.

\section{Who Should be Considered for Genetic Testing?}

Identifying women and men who should be referred for risk counseling and possible genetic testing has been controversial. Multiple risk assessment systems have been developed to quantify risk. Many of them are difficult and / or time consuming to use, and are best reserved for use in the genetic counseling process once detailed family history information has been obtained. The panelists felt that any patient with a significant risk of mutation assigned by any of these models would benefit from referral for risk counseling.

"Significant" was difficult to define, but most of the group felt that a $10 \%$ or greater risk of mutation was an appropriate definition. The following are guidelines and examples to help clinicians identify candidates for referral:

1. Early age onset of breast (or ovarian) cancer in the index patient. The panelists thought that breast cancer before age 40 was a firm criterion for referral.

2. Multiple affected relatives. Two or more affected relatives in a single bloodline before age 50, including the proband, or three or more relatives with one of the index cancers in a single bloodline should generate a referral. However, this should be modified by the population frequency of a given cancer, and the ages of onset within the family. Two postmenopausal breast cancers in one family are not unusual, but two ovarian cancers are much less likely to occur by chance. Hence, mutation carriers are more likely in a family with two ovarian cancers or two early onset breast cancers more so than one with three late onset breast cancers.

3. Multiple primary cancers in an individual, particularly breast and ovarian cancer.

4. Male breast cancer - all men with breast cancer should be referred for testing,

5. Pathology considerations. Patients with medullary or "pseudo-medullary" breast cancer are more likely to be BRCA1 carriers and should be referred. Those with triple negative breast cancer (ER, PR, and HER-2 negative) are also more likely to have a BRCA1 mutation; they also should be tested, especially if under age 50 or if associated with a family history of breast cancer.

6. Newly diagnosed breast cancer patients of Ashkenazi Jewish descent, even without any of the other criteria, if the results of testing might change management.

7. Family history of ovarian cancer in the absence of other family history of malignancy if the patient is a member of an ethnic group with a known founder mutation.

8. Known breast cancer mutation in a first- or second-degree relative, on either side.

9. The occurrence of breast cancer in the index patient and a family history of prostate, thyroid cancer, sarcoma, endometrial cancer, adrenocortical cancer, brain tumor, or pancreatic cancer. The panel thought that this was a much looser criterion than the preceding factors. 
The history of prostate cancer in a first-degree relative of a patient with breast cancer at any age also generated discussion, but no final recommendation was made.

\section{Barriers to Risk Counseling and / or Genetic Testing Reimbursement for Counseling Services and Genetic Testing}

The panelists all expressed their concern about inadequate reimbursement for genetic testing and counseling. In most of the European countries, the testing, when indicated, is usually covered by national health plans, but the counseling is not (except in the UK). In the USA, reimbursement for testing is an individual issue depending upon the individual's health insurance and / or state Medicaid programs for the uninsured. For reimbursement by Medicare in the USA, the criteria include pre-and post-test counseling by a "qualified and appropriate trained individual," but without further definition. Genetic counseling is considered a "screening service" by Medicare and may not be reimbursable. Most academic centers pro- vide genetic counseling at no or very little cost to the patient. This is not a sustainable model. Most health insurance plans do not reimburse visits to a genetic counselor unless he / she is a physician, who then must bill for a low-level office visit, not a counseling session. Insurance carriers do not usually reimburse genetics counselors because they are not recognized as ancillary providers, such as nurse practitioners or physicians' assistants. The group unanimously agreed that federal or state-wide licensing programs for genetic counselors was mandatory to permit reimbursement for this grossly undervalued but highly essential service.

\section{Fear of Discrimination}

Patient (and physician) fears of genetic discrimination by health insurers have sometimes interfered with women's pursuit of testing. In the USA, several states have already made genetic discrimination illegal, and two identical bills, HR-493 and S-398, the Genetic Information Non-Discrimination Act, banning genetic discrimination have passed both houses of the Congress. When enacted, this law will protect individuals from genetic discrimination with respect to health insurance and employment. To be made law, the House and Senate versions must be reconciled, and the president must sign the final version. President Bush has promised to do so when it is presented to him.

\section{Who Should Screen and Who Should Counsel?}

Although many high quality risk assessment pro- grams exist, they are insufficient to care for all the existing high risk patients. Access to counseling centers may be difficult because of backlogs of several months. To manage the patient load more effectively, the counseling process must be more efficient, with many more trained counselors.

A typical risk counseling session takes at least 1 hour, and pregenetic testing counseling requires an additional 2-3 hours. Much of the session involves taking a complete family history and determination of risk factors, followed by data entry into pedigree drawing software to produce a quantitative risk analysis, plus the time to complete the medical record. It is hopeful that innovative software (e.g., Hughes- RiskApps.com) will fulfill the promise of computers decreasing workload by reusing previously collected family history and risk factors and accomplishing these tasks expeditiously and accurately.

The panel was enthusiastic about the development of new and innovative ways to improve counseling efficiency and access, such as phone counseling and internet based data collection. However, the panel believes these approaches should be used cautiously and be vetted as safe and appropriate before they are used widely. 


\section{Who Can Counsel?}

The panelists agreed that counseling should be undertaken by trained, experienced individuals, but there was little agreement on defining adequate preparation. In general, all concurred that a committed clinician, genetic counselor, nurse, nurse practitioner or other medical professional, also able to provide the elements of informed consent required prior to genetic testing as delineated by ASCO, could provide a high quality service. Hospitals or practices lacking a committed individual should refer elsewhere.

The panel strongly disapproved of current internet based advertising for home-based testing with telephone counseling before and after the testing process. The safety and efficacy of these approaches have not been established. Similarly, the group frowned upon direct advertising to primary care physicians by commercial laboratories. These suggestions should not be inferred to limit the patient's primary physician, who has the interest and expertise, and who knows the patient, from ordering genetic testing so long as there is appropriate counseling and informed consent.

\section{Timing}

Another major concern of the panelists was the delay from blood draw to delivery of results. This may take weeks, even months, which, for the patient who begins the process after a diagnosis of breast cancer, as many do, is untenable. The panelists strongly endorsed urgent genetic counseling and testing for selected newly diagnosed breast cancer patients. As more counseling centers become available, this should become less of an issue. Part of the informed consent for all of these patients beginning their risk assessment after a diagnosis of breast cancer is their understanding that a reasonable delay in reporting results is not harmful to their outcome. Currently and until testing results are more rapidly reported, preoperative chemotherapy in selected patients allows systemic treatment to be initiated and completed while the results are pending. Surgical treatment can then be based upon the results of genetic testing.

\section{Management of The Patient with a Mutation}

Current genetic testing includes full sequence testing in the non-Ashkenazi patient. In Ashkenazim, testing for the three common founder mutations is performed first, and only if negative should full sequence testing be completed. When the test is negative or if one of the known mutations is detected, management is reasonably established. However, the management of individuals with VUS is not clear. Currently, the only recommendations made in this situation should be careful surveillance based upon the factors that led to the genetic testing. Carriers should be advised of the findings, and recommendations for management should be directed by a physician knowledgeable about the implications and lifetime risks of both breast and ovarian cancer (Table 4). The genetics professional providing the pre- and post-testing counseling should share the results of the testing with the patient but not make specific recommendations, unless that person is a trained oncologist. Management will differ based upon a number of variables, for example, a patient with newly diagnosed breast cancer who needs to decide about treatment. Additionally the patient should be made aware of the likelihood of the same mutation being present in children or siblings, so they can be appropriately informed. Already noted previously is the ethical and legal dilemma surrounding the "need to know" for the proband's family.

For BRCA1 or BRCA2 mutation carriers, there are really only two major choices with respect to management of each organ at risk, namely careful, lifetime follow-up, with or without chemo-prevention agents, or risk reduction surgery. Regardless of the initial management, continued lifetime follow-up is essential. 


\section{Management of Breast Cancer Risk}

The most careful or frequent nonsurgical follow-up cannot guarantee that a lethal breast cancer will not occur, so that the panel endorsed bilateral total mastectomy ( \pm reconstruction) as an almost certain technique of breast cancer prevention. The major question was timing, i.e., at the time the mutation was con- firmed or at some time in the future, the date to be chosen by the patient. Most panelists felt that surgery was best performed in the late 1930s, and should not be delayed past mid-1950s. Reconstruction was stressed as available to virtually all patients. Nipple-sparing mastectomy was addressed; all agreed that this innovation should be studied selectively. It was not to be used currently by the "occasional" breast surgeon. Although potentially as effective as total mastectomy in reducing risk, critical comparative studies comparing total mastectomy and nipple-sparing mastectomy are required before recommending this option.

The controversial role of axillary sentinel lymph node biopsy (SLNB) in mutation carriers undergoing prophylactic mastectomy was addressed because of the unexpected detection of invasive cancer in these specimens and the technical difficulty of SLNB after latissimus flap reconstruction. The almost unanimous view of the panel was that a negative MRI prior to surgery made the likelihood of an unexpected invasive cancer so low that routine SLNB was not justified.

For patients who retain their breasts, semiannual clinical examination should be supplemented by annual mammography and annual breast MRI, scheduled alternately at 6month intervals. The role of tamoxifen to reduce risk in women who retain their breasts was discussed. Lack of data prevented a substantive recommendation beyond a need to discuss the pros and cons with each patient.

Women should be aware that premenopausal prophylactic oophorectomy reduces the lifetime risk of breast cancer by as much as $50 \%$ in women with either BRCA mutation, even if HRT is used. That diminution in risk might affect a decision about prophylactic mastectomy.

\section{Management of the Mutation Carrier after the Diagnosis of Breast Cancer}

Despite the unanimous preference of the panel that every attempt be made to identify mutation carriers prior to their diagnosis, many patients learn of their high risk status only then. The goal should be the identification of all high risk women before they develop cancer. When breast cancer is confirmed, if the patient falls into one of the previously mentioned categories of increased risk, genetic testing can proceed prior to a definitive therapeutic decision. The panel unanimously agreed that the newly diagnosed breast cancer patient who proves to be a mutation carrier can be safely treated by breast conservation, expecting about the same in-breast recurrence rate as a noncarrier within

5 years, though likely having a higher rate of in-breast recurrence after about 5-10 years. Bilateral breast MRI should be performed prior to any surgery to identify multicentric disease or occult contralateral cancer, which may affect the patient's decision.

Because of the high risk of subsequent contralateral breast cancer, bilateral mastectomy should be dis- cussed, but the decision is not an urgent one. Several options are available, including preoperative chemo- therapy without further surgery while genetic testing proceeds, or local excision of the primary tumor with or without chemotherapy. After these have been completed, and after the results of genetic testing are avail- able, but before radiation, a decision can be made about definitive surgery. To radiate the patient and then perform mastectomy would be redundant, unreasonable, and may limit reconstructive 
options. The detection of an invasive cancer would generally mandate ipsilateral axillary staging (SLNB) as part of the treatment regardless of the final surgical decision. This procedure may be accomplished separately while awaiting the results of genetic testing, if the patient so desires.

The panelists were concerned about the role of prophylactic mastectomy in the patient with advanced breast cancer. A patient with multiple positive nodes will derive little benefit from a contralateral prophylactic mastectomy if her life expectancy is limited. The group stressed the need for accurate staging prior to undertaking contralateral mastectomy in these patients.

The group also agreed that, after the diagnosis of breast cancer, the mutation status should not influence adjuvant therapy recommendations. As yet, response to therapy and long-term prognosis are the same for carriers and noncarriers when matched by age, stage, and tumor characteristics. Mutation carriers should receive the same adjuvant therapy, based on their age and cancer characteristics, as women without mutations.

\section{Management of Ovarian Cancer Risk}

If risk reduction surgery is not chosen, pelvic examination and measurement of serum CA-125 at 6-month intervals were recommended. Transvaginal pelvic ultrasound was suggested every 6 months, starting at age 35 or 5-10 years younger than the earliest ovarian cancer in the family, although only limited clinical trial data substantiate these recommendations, either their efficacy or their frequency.

Oral contraceptives appear to decrease the risk of ovarian cancer but may slightly increase the risk of breast cancer. Optimal duration of use or whether their use should be occasionally interrupted was not clear. Little is known about the effects of pregnancy on the natural history of breast or ovarian carcinoma in mutation carriers, but currently pregnancy is not interdicted in these women.

Oophorectomy was considered critical, as most ovarian cancers are detected when the disease is locally advanced. Breast cancer is often detected at an early stage by screening so that oophorectomy was considered more essential in these patients than mastectomy. In carriers with previously treated breast cancer, prophylactic oophorectomy should be offered despite amenorrhea from prior chemotherapy, hormone receptor status of their breast cancer, or prior naturally occurring menopause.

For mutation carriers, bilateral salpingo-oophorectomy was recommended, and the major question was the timing of this procedure. The group suggested that surgery should be considered after childbearing is completed, but the earlier, the better. Both bilateral salpingo-oophorectomy (BSO) alone and total abdominal hysterectomy (TAH) / BSO have advantages and disadvantages, so that the procedure should be decided individually. BSO is certainly the faster, less morbid approach, but the residual small portion of fallopian tube may develop cancer, and the uterus is at risk if tamoxifen is used. On the other hand, the increased cost and morbidity of TAH are not easily justified on a universal basis. The advantage of hysterectomy is avoiding the need for a progesterone derivative if HRT is used postoperatively. Regardless of procedure chosen, the surgical specimen must be examined in toto by a skilled pathologist because the rate of occult ovarian cancer and fallopian tube dysplasia is high.

BSO does not eliminate the risk of a primary peritoneal carcinoma. The mesothelium of the peritoneum and the germinal epithelium of the ovary share the same embryologic origin; therefore, the peritoneum may retain the multipotentiality that allows the development of a 
primary carcinoma microscopically resembling ovarian carcinoma. For this reason, even after BSO, women must be carefully followed by their gynecologist.

\section{Hormone Replacement Therapy / Oral Contraceptives}

Whether premenopausal women who undergo prophylactic oophorectomy should be offered hormone replacement therapy (HRT) was another contentious question. If bilateral mastectomy is performed, then this question becomes moot. The panelists favored HRT for premenopausal women, to be continued until the time at which natural menopause might occur (early fifties), with individual decisions subsequently based upon quality of life issues.

For premenopausal women undergoing oophorectomy but not undergoing prophylactic mastectomy, most of the group advocated adding HRT. They felt that it would be unconscionable to perform oophorectomy on a young woman and then commit her to premature menopause. HRT would then be continued as above, until natural menopause might occur, etc. For already postmenopausal women not undergoing mastectomy, most of the group opposed adding HRT except to address quality of life issues as well.

Published studies show a similar reduction in risk of breast cancer after oophorectomy whether HRT is given or not. All agreed that up to 5 years of HRT was without harm, but did recognize the lack of convincing evidence-based data to support either position.

\section{The "Cancer Family" Without a Mutation}

The management of the so-called "cancer family" without a documented genetic mutation requires clarification. This group includes patients with breast cancer whose family history strongly suggests that the index patient would be a mutation carrier, as well as patients without breast cancer whose family histories are rife with malignancies, including breast and / or ovary. Despite the expectation that a mutation would be detected, the results of genetic testing of patient or family member(s) are negative for BRCA1, BRCA2, and VUS. It is often even more difficult to advise these patients of the appropriate course to follow, as risk-reduction surgery, i.e., oophorectomy and / or bilateral mastectomy, would not usually be recommended. The panel advised, how- ever, the same type of nonsurgical follow-up for these individuals, i.e., annual mammography and annual breast MRI, alternating every 6 months, along with semiannual clinical breast examinations and at least yearly pelvic examinations. These patients would also be suitable candidates for tamoxifen.

\section{Follow-up}

The group unanimously concurred that lifetime follow-up was appropriate for all women with BRCA1 / 2 mutations, regardless of whether or not they choose risk reduction surgery. For those women not undergoing oophorectomy, their follow-up should be as already mentioned previously for those women awaiting the end of child-bearing for surgery. For women not choosing prophylactic mastectomy, breast surveillance should be as already discussed above. If one or the other surgical procedure is chosen, the follow-up of the other organ should be as above. Even if both target organs are removed, continued surveillance was stressed, as mastectomy is only about $95 \%$ effective at preventing breast cancer. Annual examination of the chest wall and annual pelvic examination were still recommended. The role of CA-125 determinations and / or pelvic ultrasound was unclear.

\section{Future Directions}

The panelists were aware and supportive of the increasingly valuable information from molecular and genomic profiling of breast malignancies and their enormous importance in 
the classification and management of breast cancer. Advances in the identification of genetic markers predicting the risk of breast cancer or its recurrence are exploding exponentially. This information is already having a major impact on reclassifying and managing breast malignancies, particularly for using systemic therapies. What role these may play in the assessment and management of risk remain speculative.

Very new and as yet immature data indicate that genomic rearrangements within the same genes may occasionally identify additional carriers of nonfunctional BRCA1 and BRCA2 genes missed by conventional sequencing. Additionally, breast cancers in families without BRCA1 / 2 mutations are presumably due to other as yet unidentified high penetrance genes. Other genetic transformations or low penetrance tumor susceptibility genes are likely to account for a greater proportion of familial breast cancers in patients sent for genetic testing but who prove to be non-BRCA1 / 2 mutation carriers. In the next decade, screening for combinations of high and low penetrance genes will likely permit the identification of additional groups of individuals with inherited breast cancer.

Newer functional imaging techniques are rapidly undergoing development. These hold great promise for the earlier detection of breast cancer, which may shift the risk-reduction paradigm away from prophylactic surgery toward surveillance alone, depending upon their impact on survival after detection.

\section{Final Remarks}

The faculty of this conference represented each of the major medical specialties involved in the care of breast cancer patients, and they unanimously agreed with the concept of multidisciplinary centers as the most efficient and the most effective manner in which breast cancer should be studied. As the molecular biology of breast cancer expands exponentially, the mutual interdependence of each specialty will demand their continuing collaboration and cooperation to benefit each patient.

All of the panelists agreed that the major issues in breast cancer genetics, risk and risk management had been addressed in enough detail to permit the promulgation of these guidelines for patient care. The group recognized, however, that these published proceedings are editorial opinions of the group and only partly evidence based. Therefore, they must not be construed as establishing a standard of care to which all treating physicians should adhere. Individual patient management needs to be determined based on unique patient clinical circumstances. Recommendations for genetic counseling and management of identified risk are the responsibility of individual physician(s) with the full participation of the patient. This consensus report can- not be considered to establish standards of care for all patients, nor as guidelines for diagnostic or treatment management decisions by third party payers.

\section{Appendix: The International Consensus Conference Committee}

Benjamin O. Anderson, MD, University of Washington, Seattle, WA 98195, USA; Banu K Arun, MD, MD Anderson Cancer Center, Houston, TX 77030, USA; Harry Bartelink, MD, $\mathrm{PhD}$, The Netherlands Cancer Institute, Amsterdam, 1066 CX, The Nether- lands; Philip Bernard, MD, University of Utah, Salt Lake City, UT 84112-5550, USA; Bernardo Bonanni, MD, European Institute of Oncology, Milan, Italy; Blake Cady, MD, Brown University School of Medicine, Providence, RI 02905, USA; Krishna B. Clough, MD, Paris Breast Center (Institute du Sein), 75016 Paris, France; Susan M. Domchek, MD, University of Pennsylvania, Philadelphia PA 19104-4283, USA; Carol J. Fabian, MD, University of Kansas Medical Center, Kansas City, KS 66160-7418, USA; Stephen A. Feig, MD, FACR, University of California Irvine, School of Medicine, Orange, CA 92868-3298, USA; Ian S. 
Fentiman, MD, Guy's Hospital, London SE19RT, UK; Lynn C. Hartmann, MD, Mayo Clinic Can- cer Center, Rochester, MN 55902, USA; Sylvia H. Heywang-Köbrunner, MD, Leiterin des Referenzzentrum Mammographie, 81675 München, Germany; Roland Holland MD, PhD, National Expert and Training Centre for Breast Cancer Screening, 6503 GJ Nijmegen, The Netherlands; Anthony Howell, MD, Christie Hospital NHS Trust, Manchester, UK; Kevin S. Hughes, MD, FACS, Massachusetts General Hospital, Boston, Massachusetts 02114 USA; Claudine Isaacs, MD, Georgetown University, Washington, DC 20007, USA; Daniel B. Kopans, MD, Massachusetts General Hospital, Boston, MA 02114, USA; Henry T. Lynch, MD, Creighton University School of Medicine, Omaha, NE 68178-0001, USA; Robert E. Mansel, MD, Wales College of Medicine, Cardiff, Wales, UK; Shahla Masood, MD, University of Florida Health Science Center, Jacksonville, FL 32209, USA; Juan P. Palazzo, MD, Jefferson Medical College, Philadelphia, PA 19107, USA; Peter I. Pressman, MD, Weill Medical College of Cornell University, New York, NY 10021, USA; Mark E. Robson, MD, Memorial Sloan- Kettering Cancer Center, New York, NY 10021, USA; Gordon F. Schwartz, MD, MBA, FACS, Jefferson Medical College, Philadelphia, PA 19107-4305, USA; Lawrence J. Solin, MD, University of Pennsylvania School of Medicine, Philadelphia, PA 19104, USA; Michael Untch, MD, Academic Hospital of the University Charite Berlin, 13125 Berlin, Germany; David J Winchester, MD, FACS, Northwestern University Feinberg School of Medicine, Evanston, IL 60201, USA. 
Table 1

Risk Factors

\begin{tabular}{ll}
\hline Major $(>\mathbf{2 X})$ & Minor $(\mathbf{1}-\mathbf{2 X})$ \\
\hline Mutation associated with hereditary cancer & Late or no childbirth \\
Family history & Early menarche \\
First degree $<60$ year & Late menopause \\
Chest radiation $<30$ year & HRT \\
DCIS, LCIS, ADH, ALH & Lactation (none versus any) \\
Prior breast or ovarian cancer & Postmenopausal obesity \\
Age & Sedentary lifestyle. \\
& second and third degree relatives \\
& Alcohol, smoking \\
& Hi socio-economic status \\
& Vitamin D deficiency \\
\hline
\end{tabular}

DCIS, ductal carcinoma in situ; LCIS, lobular carcinoma in situ; ADH, atypical ductal hyperplasia; ALH, atypical lobular hyperplasia. 


\section{Table 2}

Major Factors: Absolute Risk per Year

\begin{tabular}{ll}
\hline BRCA 1/2 >age 30 & $2-3 \%$ \\
DCIS & $1-2 \%$ \\
LCIS & $1 \%$ \\
Atypia + family HX & $1 \%$ \\
Atypia alone & $0.5 \%$ \\
Prior invasive cancer & $0.75 \%$ other breast \\
Age $>60$ & $0.33 \%$ \\
\hline
\end{tabular}

DCIS, ductal carcinoma in situ; LCIS, lobular carcinoma in situ. 


\section{Table 3}

Major Factors \& Relative Risk

\begin{tabular}{ll}
\hline Factor & Relative risk (RR) \\
\hline Age (30 versus 60) & 10 \\
Intraepithelial neoplasia (LCIS, ADH, ALH) & $2-10$ \\
Prior breast/ovarian cancer & $2-10$ \\
First-degree relative <age 60 at diagnosis & $2-3$ \\
Highly penetrant mutations responsible for hereditary breast cancer & $10-20$ \\
Chest radiation, <age 30 & $5-20$ \\
\hline
\end{tabular}

LCIS, lobular carcinoma in situ; ADH, atypical ductal hyperplasia; ALH, atypical lobular hyperplasia. 
Table 4

Cumulative risks of breast \& ovarian cancer for women with $B R C A 1 / 2$ mutations *

\begin{tabular}{lrrrrr}
\hline & \multicolumn{2}{c}{ Breast cancer } & & \multicolumn{2}{c}{ Ovarian cancer } \\
\cline { 2 - 3 } Risk by age \% & BRCAI & BRCA2 & & BRCA1 & BRCA2 \\
\hline 30 & 3 & 0 & $<2$ & 1 \\
40 & 21 & 17 & 5 & 2 \\
50 & 39 & 34 & 20 & 10 \\
60 & 58 & 48 & $40-50$ & 18 \\
70 & 69 & 74 & $50-60$ & 18 \\
80 & 81 & 85 & $>60$ & 23 \\
Lifetime & 85 & 85 & 62 & 25 \\
Male carriers & 6 & 7 & & \\
Lifetime risk & & & & \\
\hline
\end{tabular}

Breast cancer: general population risk: females, $12.5 \%$; males, $<1 \%$.

Ovarian cancer: general population risk: $1-2 \%$.

Modified from: King MC, Marks JH, Mandell JB, et al: Breast \& Ovarian Cancer Risks Due to Inherited Mutations in BRCA1 and BRCA2. Science, 2003, 302: 643-646. 